\title{
Evaluation of effects of two dexamethasone formulations in impacted third molar surgeries*
}

\author{
Avaliação dos efeitos de duas formulações de dexametasona em cirurgias de terceiros molares \\ inclusos
}

Marsal Moretto Noboa'1, Juliana Cama Ramacciato², Rubens Gonçalves Teixeira'1, Carllini Barroso Vicentini³, Francisco Carlos Groppo ${ }^{4}$, Rogério Heládio Lopes Motta²

${ }^{*}$ Recebido da Faculdade São Leopoldo Mandic, Campinas, SP, Brasil.

\section{ABSTRACT}

BACKGROUND AND OBJECTIVES: Submucosal dexamethasone injection directly in the surgical area has been used in different dental procedures, but there are still few studies evaluating its efficacy as compared to oral route for impacted third molar surgeries. So, this study aimed to evaluate postoperative pain, edema and trismus after impacted third molar surgeries using oral or submucosal local injection of dexamethasone.

METHODS: This was a prospective, controlled, crossover and randomized study involving 36 patients with indication of lower third molar surgeries, who were randomly distributed in two groups: group A - submucosal local injection of dexamethasone ( $4 \mathrm{mg} / 1 \mathrm{~mL}$ ) after local anesthesia, and group B - oral dexamethasone tablet $(4 \mathrm{mg})$ one hour before procedure. Edema and trismus were clinically evaluated in the postoperative period and in the $1^{\text {st }}$, $2^{\text {nd }}, 3^{\text {rd }}$ and $7^{\text {th }}$ postoperative days. Patients were oriented to record pain intensity in the visual analog scale in periods zero (preoperative), 1h, 2h, 4h, 12h, 1 day, 2 days and 3 days and one week after surgery. Data were submitted to statistical analysis with significance level of $5 \%$.

RESULTS: There were no significant differences in surgical time with regard to operated sides $(\mathrm{p}=0.4)$. Edema and trismus values were not statistically different between observed groups ( $p>0.05)$. Mean pain values recorded in the visual analog scale were not statistically different between groups and patients have not reported ma-

1. Faculdade São Leopoldo Mandic, Campinas, São Paulo, SP, Brasil.

2. Faculdade São Leopoldo Mandic, Área de Farmacologia, Anestesiologia e Terapeutica, Campinas, SP, Brasil.

3. Faculdade Instituto Tocantinense Presidente Antonio Carlos, Departamento de Cirurgia, Porto Nacional, TO, Brasil.

4. Faculdade de Odontologia de Piracicaba FOP-UNICAMP, Área de Farmacologia, Anestesiologia e Terapêutica, Piracicaba, SP, Brasil.

Apresentado em 18 de fevereiro de 2014.

Aceito para publicaçáo em 13 de junho de 2014.

Conflito de interesses: não há.

Endereço para correspondência:

Rogério Heládio Lopes Motta

Rua José Rocha Junqueira, 13

13045-755 Campinas, SP, Brasil.

E-mail: rogeriomotta@yahoo.com

(C) Sociedade Brasileira para o Estudo da Dor jor postoperative discomfort and had no need to prolong analgesic medication ( $\mathrm{p}>0.05)$.

CONCLUSION: Both dexamethasone administration routes were effective to control pain, edema and trismus after lower third molar surgeries, presenting similar results.

Keywords: Dexamethasone, Edema, Oral surgery, Pain.

\section{RESUMO}

JUSTIFICATIVA E OBJETIVOS: A utilização da injeção submucosa de dexametasona diretamente na área cirúrgica tem sido realizada em vários procedimentos odontológicos, mas ainda são escassos os estudos que avaliaram a sua eficácia em comparação com a via oral para exodontias de terceiros molares inclusos. Desta forma, o objetivo do presente estudo foi avaliar a dor, edema e trismo no pós-operatório de cirurgia de terceiros molares inclusos utilizando-se dexametasona, por via oral, ou por injeção local submucosa.

MÉTODOS: Estudo prospectivo, controlado, cruzado e randomizado envolvendo 36 pacientes com indicação cirúrgica de terceiros molares inferiores que foram divididos em dois grupos: grupo A injeção local submucosa de dexametasona $(4 \mathrm{mg} / 1 \mathrm{~mL})$ após a anestesia local e grupo B - 1 comprimido de dexametasona ( $4 \mathrm{mg}$ ), por via oral, uma hora antes do procedimento. Edema e trismo foram avaliados clinicamente no pré-operatório, $1^{\circ}, 2^{\circ}, 3^{\circ}$ e $7^{\circ}$ dia de pós-operatório. Os pacientes foram orientados a registrar a intensidade de dor na escala analógica visual nos períodos zero (pré-operatório), 1h, 2h, 4h, 12h, 1 dia, 2 dias, 3 dias e uma semana após as cirurgias. Os dados obtidos foram submetidos a análise estatística com nível de significância de 5\%.

RESULTADOS: Não revelaram diferenças significativas no tempo cirúrgico em relação aos lados operados $(\mathrm{p}=0,4)$. Os valores de edema facial e trismo não demonstraram diferenças estatisticamente significativas entre os grupos observados $(p>0,05)$. Os valores médios de dor registrados na escala analógica visual não mostraram diferença estatística significativa entre os grupos e os pacientes não relataram grande desconforto pós-operatório e não necessitaram prolongar o fármaco analgésico ( $\mathrm{p}>0,05)$.

CONCLUSÃO: As duas vias de administração da dexametasona mostraram-se eficazes no controle da dor, edema e trismo após cirurgias de terceiros molares inferiores, apresentando resultados semelhantes.

Descritores: Cirurgia bucal, Dexametasona, Dor, Edema. 


\section{INTRODUÇÃO}

A exodontia de terceiros molares impactados envolve trauma para os tecidos moles e ósseos e podem resultar em dor considerável, edema e trismo. Esses eventos fisiopatológicos pós-operatórios estão associados à liberação de mediadores inflamatórios resultantes do metabolismo do ácido araquidônico, o que pode causar desconforto para o paciente e afetar sua qualidade vida ${ }^{1-3}$.

Muitos profissionais têm enfatizado a necessidade de um melhor controle do processo inflamatório no pós-operatório desses procedimentos cirúrgicos, e vários fármacos têm sido propostos nesse contexto. Os corticosteroides podem inibir o início da síntese de mediadores inflamatórios e são considerados fármacos potentes para o controle da dor, edema e trismo ${ }^{4-7}$.

A dexametasona tem sido amplamente utilizada na odontologia em diferentes doses e vias de administração para diminuir o desconforto pós-operatório, e quando usada por um curto período apresenta menor interferência na quimiotaxia para os leucócitos ${ }^{8,9}$. Dentre as vias de administração, a injeção submucosa de dexametasona foi relatada em estudos prévios com efeito significativo sobre o edema no pós-operatório, mas apenas um efeito limitado em trismo e dor ${ }^{10-13}$. Apesar de haver várias investigaçóes científicas acerca deste assunto, ainda não há um consenso no que diz respeito à seleção de pacientes, dosagem, tempo e via de administração dos corticosteroides. A escolha do fármaco para controle das sequelas pós-operatórias em cirurgia bucal é normalmente apoiada em experiência profissional e preferências pessoais, e nesse sentido os estudos clínicos cruzados e randomizados são importantes para esclarecer e orientar as melhores escolhas terapêuticas ${ }^{14}$.

O objetivo deste estudo foi comparar a eficácia da dexametasona administrada por via oral ou por injeção submucosa em cirurgias de terceiros molares inclusos.

\section{MÉTODOS}

Trata-se de estudo prospectivo, cruzado e randomizado. Foram selecionados 36 voluntários de ambos os gêneros, com idade entre 18 e 25 anos e sem alteraçóes sistêmicas que fossem contraindicaçóes para os procedimentos cirúrgicos. Todos os voluntários apresentavam indicação de exodontia bilateral de terceiros molares inferiores com padrão de inclusão semelhante, classificados em Classe I ou II e em posição $\mathrm{B}$, de acordo com Pell e Gregory ${ }^{15}$ e inclusão vertical ou mesioagulada, de acordo com Winter ${ }^{16}$. Na consulta inicial os pacientes foram avaliados de acordo com uma ficha clínica na qual foram anotados dados de identificação do paciente, história médica e odontológica, resultado dos exames pré-operatórios (hemograma e coagulograma), data e horário da cirurgia, tempo operatório, lado operado na sessão, quantidade de tubetes anestésicos usados e via de administração da dexametasona usada na sessão $8,12,14,17,18$.

\section{Randomização e grupos de estudo}

Para um correto controle dos pacientes e confiabilidade dos resultados foi proposto um ensaio clínico randomizado para o lado operado e terapêutica empregada, baseado nos itens de 8-10 do checklist de 2001 da The Cochrane Collaboration (Oral Health Group, University of Manchester, UK), que determina o método aleatório de geração da sequência, ocultação da legenda dos grupos e cegamento dos envolvidos ${ }^{19-21}$. Foram propostos dois protocolos farmacológicos para controle da dor e edema pós-operatórios: Grupo A - injeção local submucosa de $1 \mathrm{~mL}$ de dexametasona $4 \mathrm{mg} / \mathrm{mL}$ (Decadron; Aché Laboratórios Farmacêuticos S.A., Guarulhos, SP, Brasil) imediatamente após a anestesia por bloqueio locorregional; Grupo B - 1 comprimido de dexametasona 4mg (Decadron, Aché Laboratórios Farmacêuticos S.A., Guarulhos, SP, Brasil), por via oral, uma hora antes do procedimento.

\section{Procedimentos cirúrgicos}

Cada voluntário foi submetido a dois procedimentos cirúrgicos realizados pelo mesmo cirurgião com experiência comprovada e com intervalo de 21 dias entre a primeira e a segunda cirurgia ${ }^{22}$. Este estudo cruzado determinou que em cada procedimento cirúrgico o mesmo paciente deveria receber um dos tratamentos propostos para cada lado operado - dexametasona $(4 \mathrm{mg} / \mathrm{mL})$ por injeção submucosa em região vestibular do terceiro molar incluso ou dexametasona (4mg) por ingestão via oral de um comprimido.

Em cada procedimento cirúrgico os pacientes foram orientados a realizar bochecho vigoroso com solução de clorexidina a $0,12 \%$ (Proderma, Piracicaba, SP, Brasil) por um minuto, passaram por antissepsia extrabucal com solução de clorexidina a 2\% (Proderma , Piracicaba, SP, Brasil) e receberam recomendaçóes pós-operatórias de dieta, repouso e higiene bucal ${ }^{23}$.

Para a realização da exodontia dos dentes foram seguidos, rigorosamente, todos os princípios de técnica cirúrgica e assepsia. Foi realizado bloqueio anestésico dos nervos alveolar inferior, lingual e bucal com lidocaína a 2\% com epinefrina 1:100.000 (Alphacaine, Rio de Janeiro, Brasil ${ }^{24}$ e nas cirurgias do grupo A, logo em seguida foi administrada a dexametasona, por via subcutânea no lado previsto pela randomização. A incisão sulcular foi realizada com lâmina de bisturi no 15 (MedGoldman', São José, Brasil) e após descolamento foi obtido um retalho mucoperiósteo. A ostectomia e odontossecção foram realizadas com instrumento rotatório com irrigação fisiológica estéril abundante. Para a sutura foi usado o fio nylon 3.0 (Polysuture', São Sebastião do Paraíso, Brasil).

Após a realização do procedimento cirúrgico, todos os pacientes receberam um frasco com 8 comprimidos de paracetamol (750mg) (Tylenol', Johnson \& Johnson do Brasil Indústria e Comércio de Produtos para Saúde Ltda., São José dos Campos, SP, Brasil) e foram instruídos a tomar um comprimido a cada 6 h por dois dias. No caso de necessidade de prolongar a analgesia os voluntários foram orientados a anotar a quantidade e horários do uso adicional do analgésico.

\section{Avaliaçáo do edema e trismo}

Essa etapa foi realizada nos momentos pré-operatório, $1^{\circ}, 2^{\circ}, 3^{\circ} \mathrm{e}$ $7^{\mathrm{o}}$ dias de pós-operatório por medidas lineares. $\mathrm{O}$ edema foi medido entre o tragus e a asa do nariz e entre o tragus e a comissura labial, do lado operado, com o uso de fio seda 2.0. O trismo foi avaliado pela distância interincisal, tomada a partir da borda incisal dos incisivos centrais superiores e inferiores do lado operado. As medidas foram verificadas com o paciente em abertura bucal máxima, utilizando um paquímetro digital calibrado (Pantec ${ }^{\circ}$, São Bernardo do Campo, Brasil) no pré-operatório, $1^{\circ}, 2^{\circ}, 3^{\circ}$ e $7^{\circ}$ dias de pós-operatório ${ }^{14}$. 


\section{Avaliaçáo da dor}

Foi utilizada a escala analógica visual (EAV) impressa em 10 folhas de um livreto contendo orientaçôes sobre o preenchimento. Cada folha desse livreto representava um momento de mensuração da dor (pré-operatório, pós-operatório imediato, 1, 2, 4, 12 horas; 1, 2, 3 e 7 dias de pós-operatório). Os pacientes foram orientados a marcar com um traço sobre a linha horizontal relacionando a intensidade dolorosa, considerando a marca zero como nenhuma dor e a marca 10 como a maior dor possível ${ }^{26}$. Os voluntários foram orientados a entregar pessoalmente o livreto com as EAV preenchidas na consulta de retorno de sete dias de pós-operatório. As suas marcaçôes foram posteriormente mensuradas por paquímetro digital calibrado (Pantec', São Bernardo do Campo, Brasil), considerando a distância da marca zero ao traço registrado pelo paciente em cada momento de medida ${ }^{27}$.

\section{Análise estatística}

Foram utilizadas técnicas de estatística descritiva através de distribuições absolutas, percentuais e métodos estatísticos inferenciais. Os testes t pareado, Levene, Shapiro-Wilks, ANOVA e Tukey foram usados com nível de significância de 5\%, sendo que os cálculos foram obtidos por meio do programa BioEstat 5.0 (Fundação Mamirauá, Belém, PA).

Este estudo foi aprovado pelo Comitê de Ética de Pesquisa em seres humanos do Centro de Pesquisas Odontológicas e Faculdade de Odontologia São Leopoldo Mandic (Processo no 2009/0110), de acordo com as normas da Resolução 196/1996. Todos os pacientes foram informados dos objetivos da pesquisa e aceitaram participar mediante assinatura do Termo de Consentimento Livre e Esclarecido (TCLE).

\section{RESULTADOS}

A análise (teste t pareado) dos momentos operatórios revelou que não houve diferenças estatisticamente significativas $(p=0,7109)$ entre o tempo operatório necessário para as cirurgias do grupo $\mathrm{B}$, medicado por via oral e do grupo A, por via parenteral. O teste de Levene mostrou que as medidas "tragus-asa do nariz" ( $p=0,9973)$, "tragus-comissura" ( $p=0,1262)$, "abertura bucal" ( $p=0,1210)$ e "dor" $(\mathrm{p}=0,0935)$ apresentaram homogeneidade, sendo que o teste de Shapiro-Wilks mostrou distribuição normal para as mesmas medidas. Assim, as medidas foram submetidas à ANOVA para medidas repetidas e ao teste de Tukey. As figuras 1 e 2 apresentam os resultados da avaliação de edema.

Com relação à medida tragus-asa do nariz, a análise dos dados revelou que não houve diferenças estatisticamente significativas entre os grupos nos tempos pré-operatório $(\mathrm{p}=1,0), 1$ dia $(\mathrm{p}=0,8141), 2$ dias $(\mathrm{p}=0,8693), 3$ dias $(\mathrm{p}=0,8610)$ e 7 dias $(\mathrm{p}=0,8508)$. Entretanto, foram observadas diferenças estatisticamente significativas entre cada tempo, tanto para o grupo $\mathrm{A}(\mathrm{p}<0,0001)$ quanto para o grupo $\mathrm{B}(\mathrm{p}<0,0001)$.

À exceção do período "3 dias" ( $\mathrm{p}=0,0690)$, não houve diferenças estatisticamente significativas $(p>0,05)$ entre os grupos nos demais tempos avaliados. Também não foram observadas diferenças estatisticamente significativas entre os períodos "pré-operatório" e "7 dias" ( $\mathrm{p}=0,3385)$; entre " 1 dia" e " 2 dias" ( $\mathrm{p}=0,3146)$; e entre " 3 dias" e "7 dias" ( $\mathrm{p}=0,2120)$ para o grupo B. As demais combinaçóes de períodos mostraram diferenças significativas $(\mathrm{p}<0,05)$ para este grupo. Para o grupo A não foram observadas diferenças estatisticamente significativas considerando os períodos "pré-operatório" e "7 dias" ( $p=0,6032)$; e "1 dia" e "2 dias" ( $p=0,6002)$; sendo que os demais apresentaram diferenças significativas $(\mathrm{p}<0,05)$.

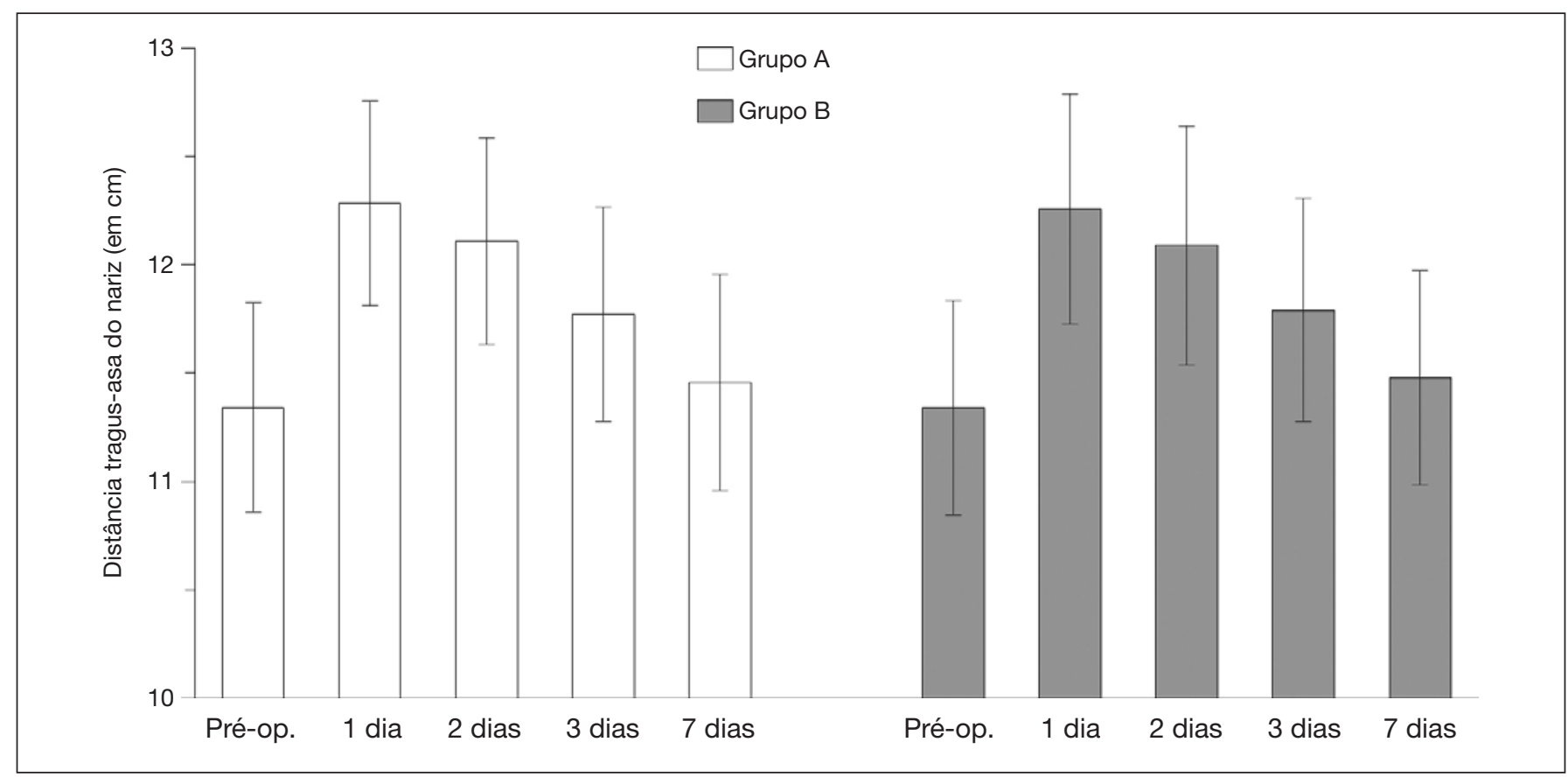

Figura 1. Distância tragus a asa do nariz (média \pm desvio padrão) em função dos tratamentos utilizados 


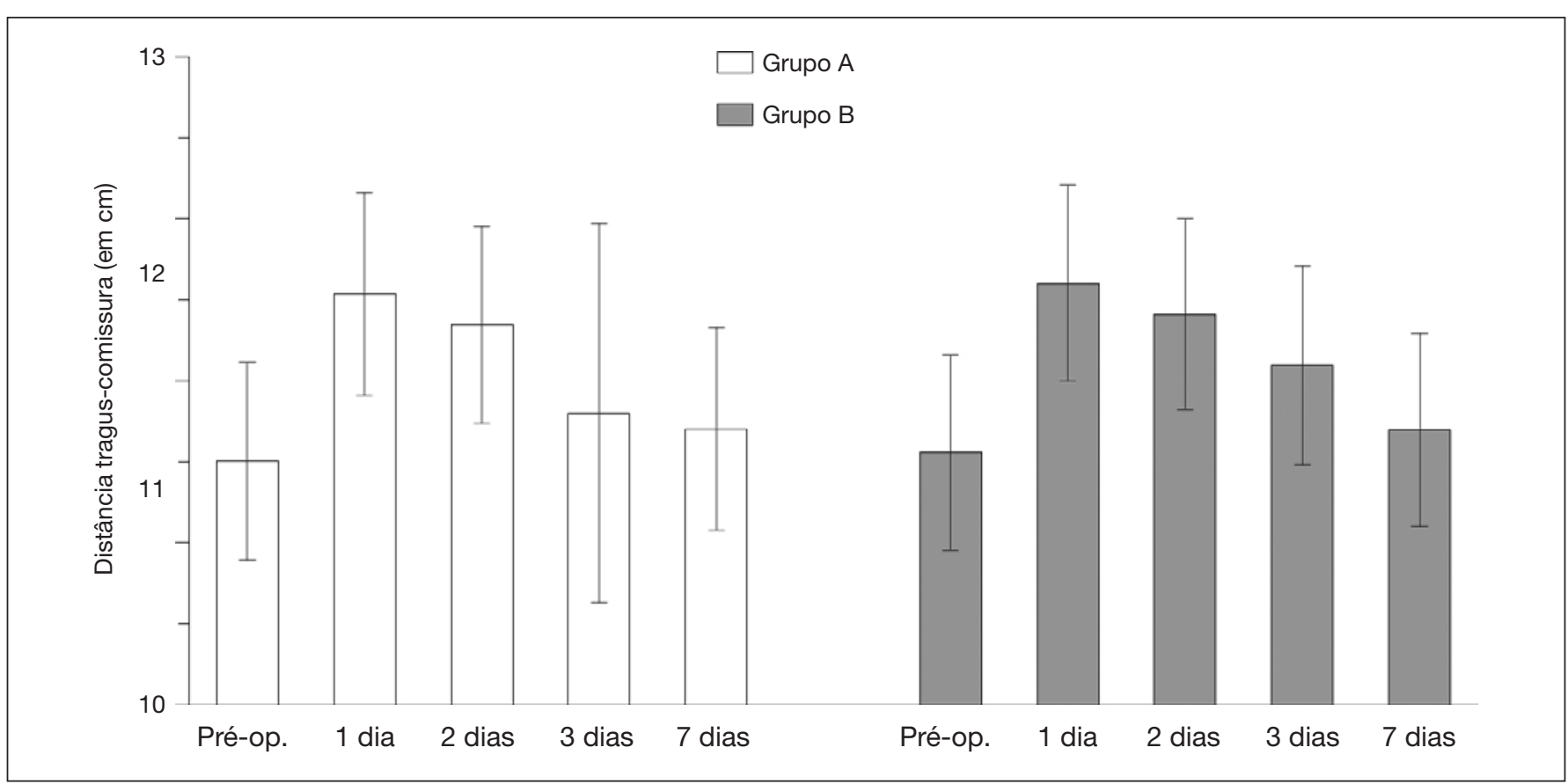

Figura 2. Distância tragus a comissura labial (média \pm desvio padrão) em função dos tratamentos utilizados

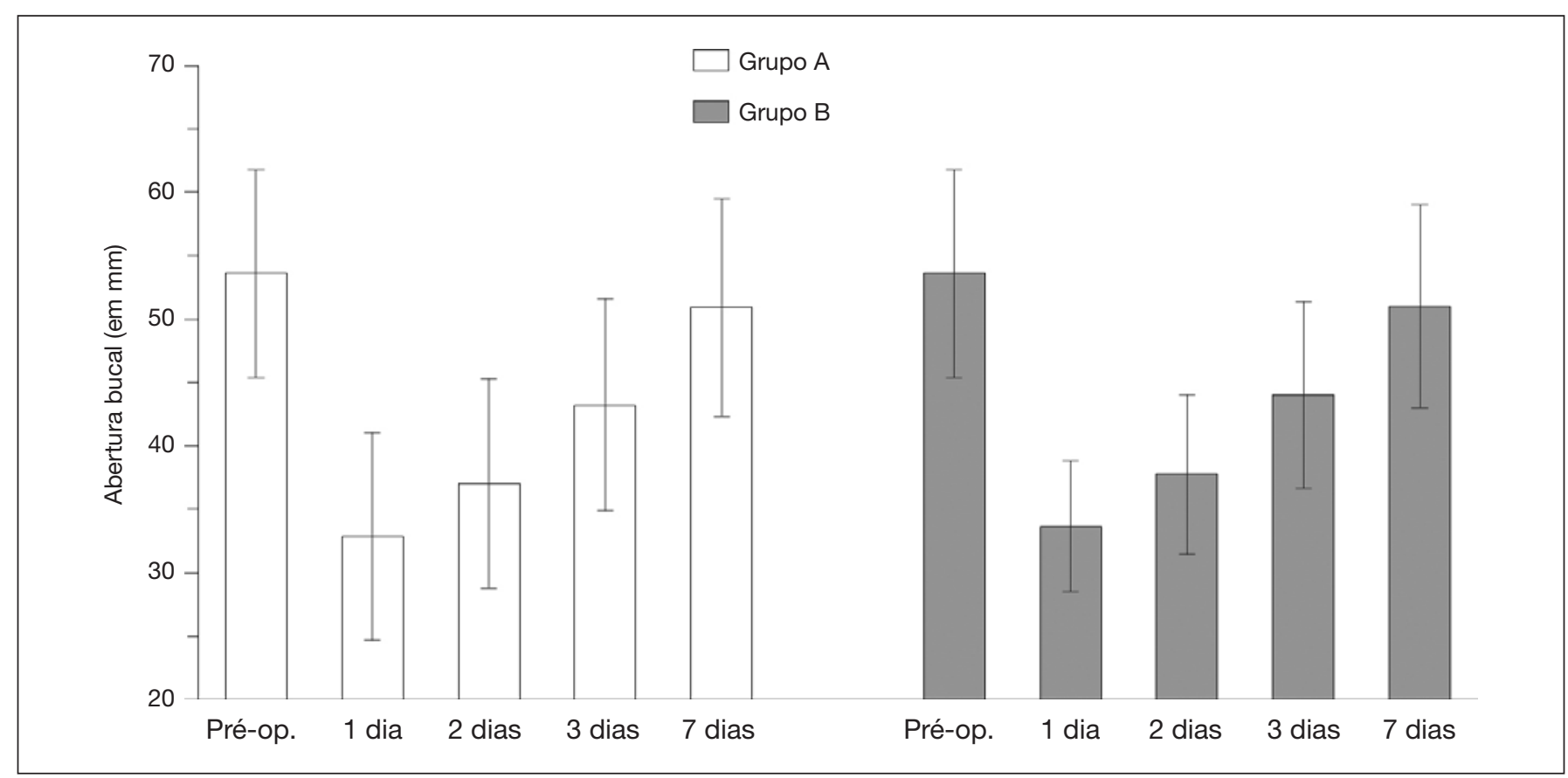

Figura 3. Valores de abertura bucal (média \pm desvio padrão) em função dos tratamentos utilizados

Com relação à abertura bucal, a análise dos dados revelou que náo houve diferenças estatisticamente significativas entre os grupos nos tempos pré-operatório $(\mathrm{p}=1,0), 1$ dia $(\mathrm{p}=0,6667), 2$ dias $(\mathrm{p}=0,6799), 3$ dias $(\mathrm{p}=0,6632)$ e 7 dias $(\mathrm{p}=0,9539)$. Entretanto, foram observadas diferenças estatisticamente significativas entre cada tempo considerando apenas o grupo $\mathrm{A}(\mathrm{p}<0,0001)$ e apenas o grupo B ( $\mathrm{p}<0,0001)$.

Com relação a avaliação da dor, a análise dos dados revelou que não houve diferenças estatisticamente significativas entre os grupos em nenhum dos tempos avaliados: pré-operatório $(\mathrm{p}=0,8981), 1$ hora $(\mathrm{p}=0,8268), 2$ horas $(\mathrm{p}=0,3254), 4$ horas $(\mathrm{p}=0,2813), 12$ horas $(\mathrm{p}=0,1978), 1$ dia $(\mathrm{p}=0,1185), 2$ dias $(\mathrm{p}=0,2180), 3$ dias $(\mathrm{p}=0,4030)$ e 7 dias $(p=0,7435)$. Entretanto, foram observadas diferenças estatisticamente significativas $(\mathrm{p}<0,0001)$ entre os períodos pré-operatórios, $1 \mathrm{~h}, 2 \mathrm{~h}, 2$ dias e 3 dias tanto para o grupo A quanto para o grupo B. Entretanto, os períodos "4h" e "12h" e "1 dia" e "7 dias" não mostraram diferenças estatisticamente significativas para ambos os grupos ( $p>0,05)$. 


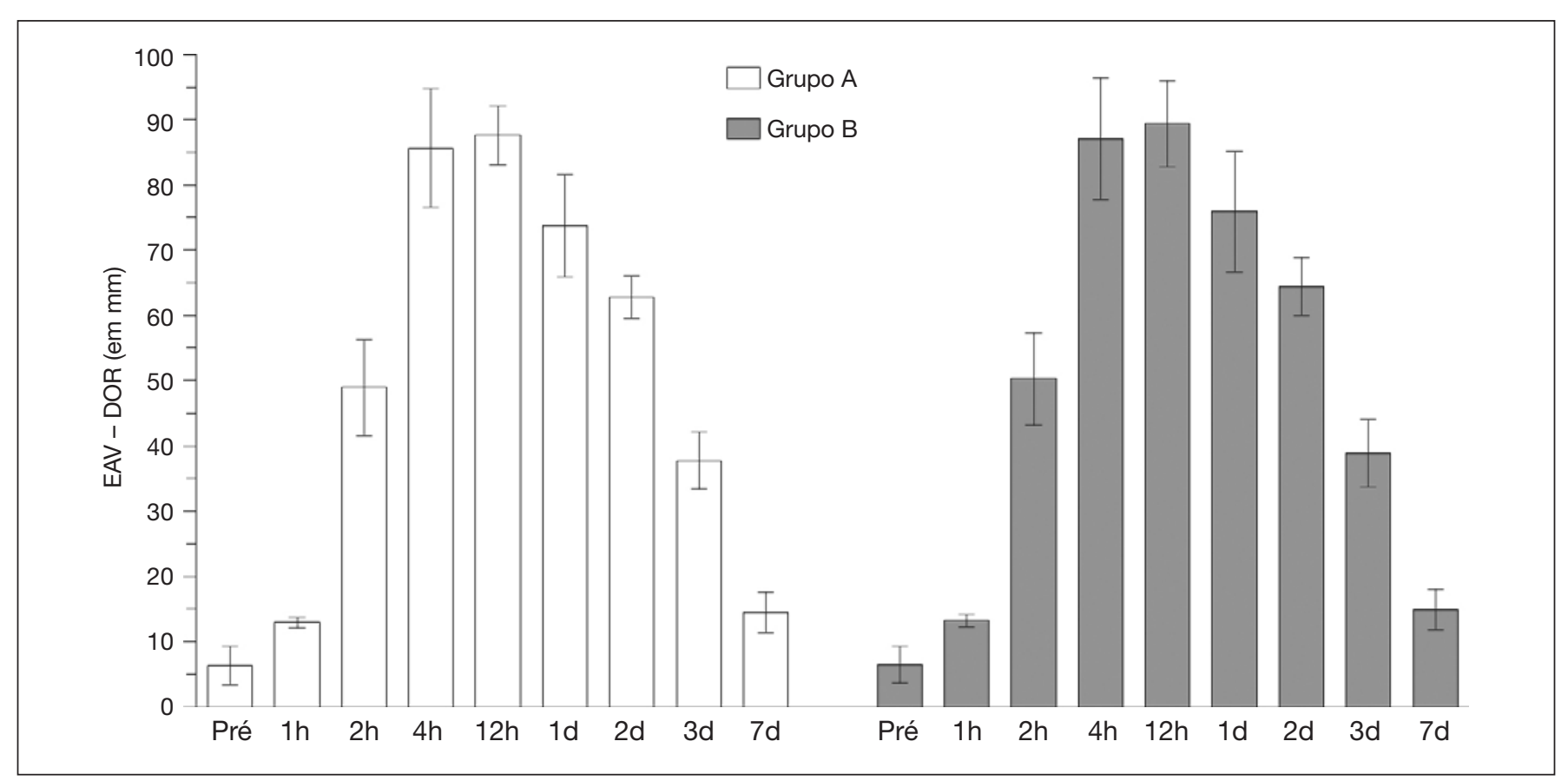

Figura 4. Médias \pm desvio padrão obtidas a partir das medidas da escala analógica visual (EAV em mm) em função dos tratamentos utilizados

\section{DISCUSSÃO}

A exodontia de terceiros molares inclusos geralmente está associada a um desconforto pós-operatório moderado a grave, mesmo quando a técnica utilizada tenha sido empregada sutilmente, conforme demonstrado na figura 4, que evidencia maior sensibilidade dolorosa nos períodos de 4, 12 e 24 h após os procedimentos ${ }^{1-3,10}$. Existe uma grande discussão sobre o melhor fármaco para amenizar o desconforto pós-cirúrgico em odontologia e dentre os fármacos de escolha, o uso da dexametasona vem sendo avaliado em diversos estudos científicos devido à sua eficácia no controle das complicaçôes inflamatórias quando comparado ao não uso ${ }^{5-7}$.

Meechan e Seymour ${ }^{28}$ estudaram as diversas complicaçóes que surgem após a cirurgia de terceiros molares impactados e concluíram que a observação dessas complicaçóes é importante para avaliar comparativamente a eficácia de uma variedade de medidas terapêuticas. Outros autores demonstraram que o procedimento cirúrgico e as observaçóes pós-operatórias imediatas constituem um modelo clínico para avaliação da eficácia de diferentes tipos de fármacos ${ }^{14,18,29,30}$. No modelo cruzado usado neste estudo, o mesmo paciente foi submetido aos dois tratamentos propostos (injeção submucosa e via oral), um em cada lado operado por ocasião da randomizaçáo. Este modelo corresponde a uma vantagem para avaliação prospectiva de farmacologia clínica, uma vez que o paciente garante a qualidade de seu próprio controle.

Alguns estudos sugerem o uso sistêmico dos corticosteroides em cirurgias de terceiros molares inclusos ${ }^{5-7,12,13}$. Markiewicz et al. ${ }^{4}$ em metanálise concluíram que a administração de corticosteroides no pré-operatório apresentou grande valor na redução dos sinais e sintomas inflamatórios pós-operatórios. Especificamente, os pacientes que receberam corticosteroides tiveram significativamente menos edema, dor e trismo pós-operatórios, tanto no início (após 1-3 dias) como no período tardio (após 4-7 dias). Apesar desses resultados, ainda existe uma falta de consenso sobre a melhor via de administração, dose, e duração do tratamento, além de diferenças nos métodos utilizados para avaliar as variáveis clínicas.

A administração por via oral de dexametasona envolve início mais tardio do efeito, fato inerente à sua farmacocinética e exige a cooperaçáo do paciente ${ }^{31}$. No entanto, constitui uma via conveniente, segura e de baixo custo. Os dados deste estudo mostraram que a dexametasona via oral foi eficaz no controle da dor e edema durante o período estudado, o que está de acordo com outros estudos similares ${ }^{7,6,11,12}$.

A injeção submucosa de dexametasona apresentou um efeito significativo sobre o edema em dois estudos anteriores sendo que ambos relataram redução significativa do edema no pós-operatório imediato em comparaçáo com os controles ${ }^{1,13}$. Os resultados deste estudo mostraram que a injeção submucosa da dexametasona produziu redução significativa do edema nos primeiros dias de pós-operatório, em acordo com os estudos anteriores ${ }^{10,13}$. Uma observação interessante nesse grupo foi a redução significativa do trismo no primeiro dia de pós-operatório, o que é semelhante ao grupo B (via oral), fato que pode ter sido resultado da concentração mais elevada de dexametasona conseguida imediatamente no local da lesão. Esses resultados adicionam mais força para o conceito de que a dexametasona administrada perto do local da cirurgia é uma forma valiosa para reduzir o edema e o trismo nesses pacientes ${ }^{5,6,12,32}$.

Os resultados do presente estudo, referentes aos testes de Levene e de Shapiro-Wilks, não mostraram diferença estatisticamente significativa entre os grupos em relação à redução da dor pós-operatória, do edema e do trismo após a exodontia dos terceiros molares. Isto corrobora com vários autores que observaram a eficácia terapêutica da administração submucosa de corticosteroides em estudos prévios, demonstrando que a injeção submucosa de dexametasona, assim 
como a administração por via oral, pode ser uma alternativa viável para procedimentos odontológicos mais invasivos ${ }^{6,10,12,13}$.

\section{CONCLUSÃO}

A administração em dose única de dexametasona por via parenteral, sob a forma de injeção submucosa, e por via oral, na forma de comprimido, demonstraram efeitos similares na redução da dor, edema e trismo após exodontias de terceiros molares inclusos.

\section{REFERÊNCIAS}

1. Majid OW. Submucosal dexamethasone injection improves quality of life measures after third molar surgery: a comparative study. J Oral Maxillofac Surg. 2011;69(9):2289-97.

2. Herrera-Briones FJ, Prados Sánchez E, Reyes Botella C, Vallecillo Capilla M. Update on the use of corticosteroids in third molar surgery: systematic review of the literature. Oral Surg Oral Med Oral Pathol Oral Radiol. 2013;116(5):e342-51.

3. Mehra P, Reebye U, Nadershah M, Cottrell D. Efficacy of anti-inflammatory drugs in third molar surgery: a randomized clinical trial. Int J Oral Maxillofac Surg. 2013;42(7):835-42.

4. Markiewicz MR, Brady MF, Ding EL, Dodson TB. Corticosteroids reduce postoperative morbidity after third molar surgery: a systematic review and meta-analysis. J Oral Maxillofac Surg. 2008;66(9):1881-94.

5. Klongnoi B, Kaewpradub P, Boonsiriseth K, Wongsirichat N. Effect of single dose preoperative intramuscular dexamethasone injection on lower impacted third molar surgery. Int J Oral Maxillofac Surg. 2012;41(3):376-9.

6. Bhargava D, Sreekumar K, Deshpande A. Effects of intra-space injection of Twin mix versus intraoral-submucosal, intramuscular, intravenous and per-oral administration of dexamethasone on post-operative sequelae after mandibular impacted third molar surgery: a preliminary clinical comparative study. Oral Maxillofac Surg. 2013;20 [Epub ahead of print].

7. Simone JL, Jorge WA, Horliana AC, Canaval TG, Tortamano IP. Comparative analysis of preemptive analgesic effect of dexamethasone and diclofenac following third molar surgery. Braz Oral Res. 2013;27(3):266-71.

8. Sotto-Maior BS, Senna PM, de Souza Picorelli Assis NM. Corticosteroids or cyclooxygenase 2-selective inhibitor medication for the management of pain and swelling after third-molar surgery. J Craniofac Surg. 2011;22(2):758-62.

9. Li J, Wang X, Zhou C, Liu L, Wu Y, Wang D, Jiang H. Perioperative glucocorticosteroid treatment delays early healing of a mandible wound by inhibiting osteogenic differentiation. Injury. 2012;43(8):1284-9.

10. Grossi GB, Maiorana C, Garramone RA, Borgonovo A, Beretta M, Farronato D, et al. Effect of submucosal injection of dexamethasone on postoperative discomfort after third molar surgery: a prospective study. J Oral Maxillofac Surg. 2007;65(11):2218-26.

11. Apiliogullari S, Yildirim G, Ataoglu H. The supraperiosteal route of dexamethasone should be considered for dental surgery. Oral Surg Oral Med Oral Pathol Oral Radiol Endod. 2010;109(2):165; author reply 165-6.

12. Antunes AA, Avelar RL, Martins Neto EC, Frota R, Dias E. Effect of two routes of administration of dexamethasone on pain, edema, and trismus in impacted lower third molar surgery. Oral Maxillofac Surg. 2011;15(4):217-23.

13. Warraich R, Faisal M, Rana M, Shaheen A, Gellrich NC, Rana M. Evaluation of postoperative discomfort following third molar surgery using submucosal dexamethasone - a randomized observer blind prospective study. Oral Surg Oral Med Oral Pathol
Oral Radiol. 2013;116(1):16-22.

14. de Sousa Santos JA, da Silva LC, de Santana Santos T, Menezes Júnior LR, de Assunção Oliveira AC, Brandão JR. Comparative study of tramadol combined with dexamethasone and diclofenac sodium in third-molar surgery. J Craniomaxillofac Surg. 2012;40(8):694-700.

15. Pell GJ, Gregory GT. Impacted mandibular third molars: classification and modified technique for removal. Dent Dig. 1933;39:e330-e8.

16. Winter GB. Principles of exodontia as applied to the impacted third molar. St. Louis: American Medical Books; 1926.

17. Graziani F, D'Aiuto F, Arduino PG, Tonelli M, Gabriele M. Perioperative dexamethasone reduces post-surgical sequelae of wisdom tooth removal. A split-mouth randomized double-masked clinical trial. Int J Oral Maxillofac Surg. 2006;35(3):241-6.

18. Laureano Filho JR, Maurette PE, Allais M, Cotinho M, Fernandes C. Clinical comparative study of the effectiveness of two dosages of dexamethasone to control postoperative swelling, trismus and pain after the surgical extraction of mandibular impacted third molars. Med Oral Patol Oral Cir Bucal. 2008;13(2):E129-32.

19. Moher D, Schulz KF, Altman D. CONSORT Statement: revised recommendation for improving the quality of reports of parallel-group randomized trials 2001. Explore (NY). 2005;1(1):40-5.

20. Siddiqi A, Morkel JA, Zafar S. Antibiotic prophylaxis in third molar surgery: A randomized double-blind placebo-controlled clinical trial using split-mouth technique. Int J Oral Maxillofac Surg. 2010;39(2):107-14.

21. Aznar-Arasa L, Harutunian K, Figueiredo R, Valmaseda-Castellón E, Gay-Escoda C. Effect of preoperative ibuprofen on pain and swelling after lower third molar removal: a randomized controlled trial. Int J Oral Maxillofac Surg. 2012;41(8):1005-9.

22. van Gool AV, Ten Bosch JJ, Boering G. Clinical consequences of complaints and complications after removal of the mandibular third molar. Int J Oral Surg. 1977;6(1):29-37.

23. Hedström L, Sjögren P. Effect estimates and methodological quality of randomized controlled trials about prevention of alveolar osteitis following tooth extraction: a systematic review. Oral Surg Oral Med Oral Pathol Oral Radiol Endod.2007;103(1):8-15.

24. Gordon SM, Chuang BP, Wang XM, Hamza MA, Rowan JS, Brahim JS, et al. The differential effects of bupivacaine and lidocaine on prostaglandin E2 release, cyclooxygenase gene expression and pain in a clinical pain model. Anesth Analg. 2008;106(1):321-7.

25. Neupert EA 3rd, Lee JW, Philput CB, Gordon JR. Evaluation of dexamethasone for reduction of postsurgical sequelae of third molar removal. J Oral Maxillofac Surg. 1992;50(11):1177-83

26. Kaczmarzyk T, Wichlinski J, Stypulkowska J, Zaleska M, Panas M, Woron J. Single-dose and multi-dose clindamycin therapy fails to demonstrate efficacy in preventing infectious and inflammatory complications in third molar surgery. Int J Oral Maxillofac Surg. 2007;36(5):417-22

27. Collins SL, Moore RA, McQuay HJ. The visual analogue pain intensity scale: what is moderate pain in millimetres? Pain. 1997;72(1-2):95-7.

28. Meechan JG, Seymour RA. The use of third molar surgery in clinical pharmacology. Br J Oral Maxillofac Surg. 1993;31(6):360-5.

29. Ong CK, Lirk P, Seymour RA, Jenkins BJ. The efficacy of preemptive analgesia for acute postoperative pain management: a meta-analysis. Anesth Analg. 2005;100(3):757-73.

30. Pektas ZO, Sener M, Bayram B, Eroglu T, Bozdogan N, Donmez A, et al. A comparison of pre-emptive analgesic efficacy of diflunisal and lornoxicam for postoperative pain management: a prospective, randomized, single-blind, crossover study. Int J Oral Maxillofac Surg. 2007;36(2):123-7.

31. Dan AE, Thygesen TH, Pinholt EM. Corticosteroid administration in oral and orthognathic surgery: a systematic review of the literature and meta-analysis. J Oral Maxillofac Surg. 2010;68(9):2207-20

32. Boonsiriseth K, Klongnoi B, Sirintawat N, Saengsirinavin C, Wongsirichat N. Comparative study of the effect of dexamethasone injection and consumption in lower third molar surgery. Int J Oral Maxillofac Surg. 2012;41(2):244-7. 